\title{
A Web-based program for coding open-ended response protocols
}

\author{
S. L. AMES \\ University of California, Los Angeles, California \\ and University of Southern California, Los Angeles, California \\ P. E. GALLAHER and P. SUN \\ University of Southern California, Los Angeles, California \\ S. PEARCE \\ University of California, Los Angeles, California \\ J. B. ZOGG \\ University of Southern California, Los Angeles, California \\ B. R. HOUSKA \\ University of California, Los Angeles, California \\ and University of Southern California, Los Angeles, California \\ B. C. LEIGH \\ University of Washington, Seattle, Washington \\ and \\ A. W. STACY \\ University of California, Los Angeles, California \\ and University of Southern California, Los Angeles, California
}

\begin{abstract}
A Web-based coding application was designed to improve coding efficiency and to provide a systematic means of evaluating responses to open-ended assessments. The system was developed for use by multiple raters to assign open-ended responses to predetermined categories. The application provides a software environment for efficiently supervising the work of coders and evaluating the quality of the coding by (1) systematically presenting open-ended responses to coders, (2) tracking each coder's categorized responses, and (3) assessing interrater consistency at any time in order to identify coders in need of further training. In addition, the application can be set to automatically assign repeated responses to categories previously identified as appropriate for those responses. To evaluate the efficacy of the coding application and to determine the statistical reliability of coding open-ended data within this application, we examined data from two empirical studies. The results demonstrated substantial interrater agreement on items assigned to various categories across free and controlled association tasks. Overall, this new coding application provides a feasible method of reliably coding open-ended data and makes the task of coding these data more manageable.
\end{abstract}

This research was supported by National Institute on Drug Abuse Grants DA12101 and DA16094 and National Institute on Alcohol Abuse and Alcoholism Grant AA12128. The authors thank Wei Sun and Juan Huang for their contributions to the development of the Web-based coding application. The Web-based coding application described in this manuscript is available to researchers free of charge. If you are interested in using the application, please contact the first author. Correspondence concerning this article should be addressed to S. L. Ames, Institute for Health Promotion and Disease Prevention Research, Department of Preventive Medicine, University of Southern California, 1000 South Fremont, Unit 8, Los Angeles, CA 91803 (e-mail: sames@usc.edu).

Note-This article was accepted by the previous editor, Jonathan Vaughan.
This article provides an overview of a newly developed Web-based program for analyzing participant-generated or open-ended data. The program is designed to exploit the advantages and limit the disadvantages of research using these types of variables. The application may be particularly valuable to research using the large sample sizes often required in studies of frequency norms in experimental psychology. This application can be applied to almost any research in which word productions need to be categorized. In experimental psychology, the application can be used for coding free and cued recall responses when the tests are scored using a gist criterion. The application 
can also be used for scoring productions in stem completion, word or picture association, and other purposes for which participant-generated words need to be scored into categories to evaluate individual or group differences. In our work, we use the coding application to categorize associates of risk behaviors (e.g., alcohol and other drug use, risky sexual behavior) that have been generated by individuals on a variety of verbal and nonverbal associative memory tasks. These coded verbatim responses can then be analyzed in prediction models of behavior. The coding program is flexible and provides a systematic means of categorizing response content and of evaluating coding quality control and interjudge reliability among even relatively untrained coders.

The coding application is also useful for survey research that elicits open-ended responses, whether the surveys are used in cognitive science, other areas of psychology, or other social sciences. In survey research, the practical use of open-ended assessments versus fixed alternative items is an important consideration for investigators. Fixed or close-ended items tend to limit participants' response choices (Bishop, Tuchfarber, \& Oldendick, 1986) and "respondents tend to confine their answers to the choices offered, even if the researcher does not wish them to do so" (Krosnick, 1999, p. 544). Alternatively, open-ended items are developed to elicit unconfined and unbiased responses and, as a result, can be more motivating to respondents. Open-ended items can be more reliable than closed-ended items (Krosnick, 1999), and they have been used as valid measures of behavior (see, e.g., Bornstein, 1999). However, fixed alternative items have had the advantage of economy: Evaluation of substantial amounts of information using such measures has traditionally been less expensive and less time consuming than open-ended testing. Survey researchers may have discounted the use of open-ended assessments for various other practical reasons, such as finding analyses of open-ended data to be too time-consuming, especially without a systematic means of reliably summarizing and categorizing qualitative responses from large samples. The present system can ease the often labor-intensive task of coding responses to open-ended questions from very large samples.

In order to improve coding efficiency, this application can be set to automatically assign repeated responses to categories previously identified as appropriate for those responses. Although there are a number of applications on the market that provide automatic coding, such as SPSS's Verbastat 7.0 (2004) and STATPAC's Verbatim Blaster (Walonick, 2004), they do not provide an environment for assessing the interrater reliability of the coding. The CDC's EZ-Text application (Carey, Wenzel, Reilly, Sheridan, \& Steinberg, 1998) does have a reliability module, but is suited for the analysis of semistructured interview data, whereas the present application is designed for coding short, open-ended responses to stimulus cues in large-scale surveys. In addition, there are various coding applications that have been developed to benefit the coding of verbal data and other audio rather than written responses (e.g., see Crutcher, 2003; James \& Sanderson, 1991; White, King, \& Duncan, 2002). As an example, the SHAPA system is an interactive verbal protocol analysis system developed to examine verbalizations. This coding system provides a flexible software environment that allows the researcher to develop a set of coding categories; code a response more than once; use alternate classification schemes; and perform summary analyses, including reliability analyses. This system, however, does not have an automatic code mode (James \& Sanderson, 1991).

The present coding application expands on many of the coding systems available in that it is designed to present open-ended responses via a Web-based interface to any number of independent coders and a supervisor. The supervisor sets the coding parameters for a particular project and monitors the quality of the coding. The system identifies raters whose coding appears to be unreliable and provides an interface for resolving coding discrepancies. The quality control features ensure that the data that are exported for further analysis are reliably coded. To date, investigators have rarely reported interrater agreement statistics in studies using word production tests such as word association. Moreover, coding procedures are rarely described in detail in written reports. Coding applications can make assumptions and coding procedures more explicit and, hence, potentially more standardized and replicable.

Following an overview of the coding application, we present findings from two studies in which we demonstrate the feasibility of reliably coding participant-generated responses with the application. We present interrater agreement statistics for the category assignments of coders on free and controlled word association tasks. The present examples of the coding application were tailored to health behavior research. The health behavior categories we defined can be easily replaced with another coding scheme defined by the user, thus making this Web-based application easily adapted for use in many areas of research requiring the coding of open-ended data.

\section{OVERVIEW OF THE BASIC CODING APPLICATION}

This application is Web based, which means that raters may code the data from any Internet-enabled computer. It was deliberately designed so that nearly any individual with minimal computer training might use the program without difficulty. Coders are provided minimal one-onone training to assist them in learning the application, and retraining is provided if needed to ensure a coder's confidence in the coding process. There are several features available in this application that make its use flexible and easily tailored to particular research aims. Features include automatic coding, coding to multiple categories, the facility to code with the stimulus cues either visible or not visible to the coder, and a number of quality control features that allow monitoring of coding quality at any time during the course of the coding project. The ability of the 
program to assist in monitoring and other coder management tasks may be one of its greatest assets.

\section{Automatic Coding}

The application is designed to separate previously coded responses from uncoded ones and can be set to automatically assign any response that has already been coded to the appropriate category. For example, if the response "draft" has been coded as an alcohol word for a specific variable, then the next time the word "draft" appears in response to that variable for that coder, it can be automatically assigned to the alcohol category and does not need to be coded again. The automatic coding option can be adjusted so that duplicate responses are autocoded only for a particular cued word or phrase, and the automatic coding can be turned off if the researcher prefers this option. If the researcher chooses to use the automatic coding option, then only those responses that have never been coded are displayed on the screen seen by the rater. Coded responses from one study can be used to facilitate coding on subsequent studies. That is, researchers can borrow coded responses from a data bank containing coded data from prior studies. For example, open-ended responses from a posttest may use codings obtained from a pretest when the responses are identical. However, unique responses to overlapping pre- and posttest items would still need to be coded.

\section{Coding to Multiple Categories}

The application also allows coders to assign ambiguous responses, or those consisting of multiple words, to more than one category. For example, a response such as "smoking and drinking" can be assigned to both the alcohol and tobacco categories. If a coder assigns this type of response (e.g., smoking and drinking) to marijuana, alcohol, and tobacco use categories, then repetitions of this response for the same variable are automatically assigned to the same multiple categories previously assigned if the system is set to automatic coding.

\section{Prompted vs. Unprompted Coding}

The coding application allows investigators to choose whether to code prompted or unprompted data. Coding with a prompt means that the cued words or phrases to which the study participants responded during the surveyed situation are visible on the coding screen at the time of coding. Alternatively, unprompted coding means that the cued words or phrases to which the participants responded are not visible on the coding screen at the time of coding. The option to choose prompted or unprompted coding is available for each dataset loaded into the application and is accessible by the coding administrator.

\section{Reliability Checks and the Consensus Function}

The program easily manages the coding of two or more raters on any set of verbatim responses. In addition to making "on-the-fly" statistical reliability analyses possible, this is done for quality control and training purposes. Comparing the coding of independent raters can reveal whether a particular coder's work is significantly different from the work of other coders, signaling a potential need for feedback or retraining. Interrater reliability statistics are based on uniquely coded responses when using the automatic coding option; that is, repeated responses that are automatically coded for a single cued word or phrase are not included in these analyses.

After two raters have completed coding a set of openended responses, a third coder may be assigned to resolve any coding discrepancies. This would be done using the coding program's consensus function. The consensus function compares the response tables for two designated coders and displays both coders' category decisions side by side on a new coding screen. Also displayed is a blank column for a third coder, who, based on the previous two sets of codes, makes a definitive category choice. The third person simply chooses one response over another and the rule basis for this choice is up to the individual researcher. An alternative option is to have a third coder independently code all responses without seeing the first two coders' responses. Consensus answers, as well as the original coders' responses, would be retained in the database.

\section{Quality Control}

Another benefit of the present coding application is an inclusive quality control process. Implementing quality control procedures helps ensure that the coded data are reliable. Data quality is assured by reviewing any of three reports that can be generated by the application: (1) responses by category, (2) coder comparison, and (3) interrater agreement analyses.

Response table review. An initial step in the quality control process is for a coding supervisor to review for each coder the Responses by Category Report, which displays an alphabetical list of all coded responses sorted by the categories to which they were assigned (see Figure 1). This report allows supervisors to review each response to determine if it was coded accurately. Supervisors can then provide feedback to coders throughout the coding process and give them a list of all responses incorrectly categorized. Responses mistakenly placed in a designated category would be recorded as inaccurately coded and corrected by the coder or a coding supervisor as necessary. The coders may simply browse their coded results and make corrections.

The Responses by Category Report can be generated in two ways. One method generates all responses coded by any one coder between any two dates. (The program retains the automatically coded repetition responses from date to date.) The other method generates all responses for a particular variable, listed by the category to which it was assigned by the coder, without considering the coding dates. The two methods facilitate a flexible review of the coders' work. Coder responses may be reviewed regularly during active coding.

Coder comparison review. The second step in the quality control process is to ensure that coders' datasets are reasonably consistent. The Coder Comparison Report 


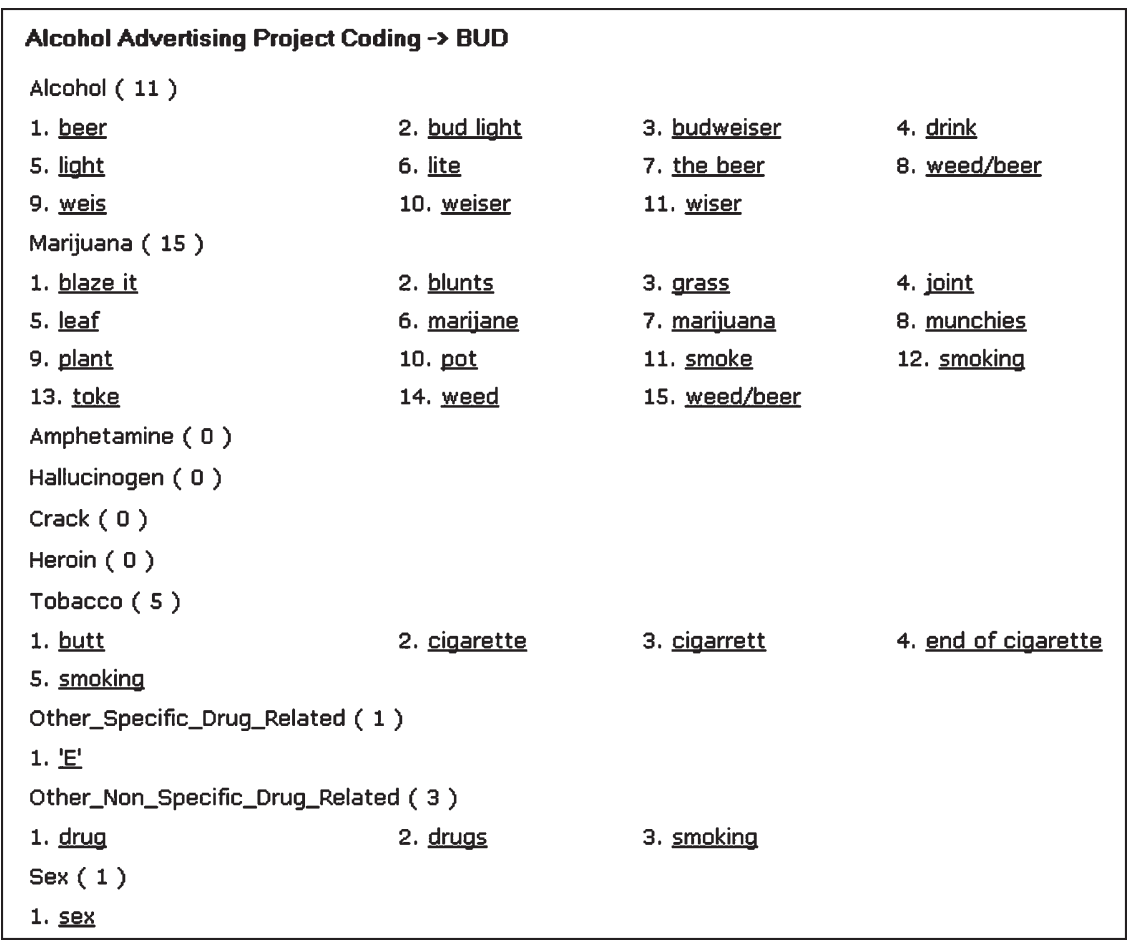

Figure 1. Example of one coder's output for the Responses by Category Report for the variable "bud." The underlines in this figure are hyperlinks back to the original coding screen for the respective items.

may be generated for this purpose (see Figure 2). This report allows a side-by-side comparison of two coders' responses assigned to a specific category, which is designated at the top of the report (for example, in Figure 2, the category is Alcohol). This format makes clear any discrepancies between coders and helps identify any nonstandard tendencies.

Multiple coded category review. Another feature of the application is the ability to print a report that includes only those responses consisting of multiple words (e.g., smoking and drinking) or ambiguous words that have been coded to more than one category. This feature allows the researcher to review these types of responses and systematically prioritize or assign responses to the behavioral categories that can be included in subsequent analyses without violating independence assumptions. For some researchers, being able to code items to multiple categories is advantageous because some participant-generated responses may be ambiguous or include more than one behavior of interest. By using a function that limits coding to a chosen number of categories, the researcher may decide whether or not all responses can be coded into only one or into more than one category.

Interrater agreement analyses. An Interrater Agreement Report provides a reliability statistic as a supplement to the Coder Comparison Report. The Interrater Agreement Report compares two coders' work for a selected variable and generates a Cohen's kappa coefficient (i.e., chance-corrected agreement on category assignment be- tween two coders) for as many categories as the researcher desires. Along with the Coder Comparison Report, the Interrater Agreement Analyses Report helps determine the extent of coder agreement and whether coders can reliably assign open-ended responses to the designated behavior categories. If these reports do not reflect acceptable agreement between two coders, and the disagreement is clearly due to coder error as opposed to genuine disagreement in judgment, coders can be retrained until they can accurately code the responses. Interrater agreement analyses are based on uniquely coded responses, whether the autocode mode is on or off. In other words, only the independent judgments made by the coders (and not automatically coded repetitions) are included in computed reliability reports.

\section{TECHNICAL FEATURES}

The coding program is housed on a Linux server and is implemented with PHP and MySQL. ${ }^{1}$ It is optimized for use with the Microsoft Internet Explorer browser and may be accessed at http://ipr1.hsc.usc.edu/codedemo/. The program is password protected, and visitors may log on using "admin" as both the username and password to test the application. There are extensive help files available online for both coders and supervisors.

The program includes functions to (1) define and set up the data structure on the server, (2) upload the to-becoded dataset, (3) define the coding scheme, (4) define 


\begin{tabular}{|c|c|}
\hline \multicolumn{2}{|c|}{ Alcohol Advertising Project Coding -> category } \\
\hline Alcohol & \\
\hline Rater A ( 16) & Rater B (11) \\
\hline \multicolumn{2}{|l|}{ 1: air bud } \\
\hline 2: beer & 2: beer \\
\hline \multicolumn{2}{|l|}{ 3: bud } \\
\hline 4: bud light & 4: bud light \\
\hline 5: budweiser & 5: budweiser \\
\hline 6: drink & 6: drink \\
\hline \multicolumn{2}{|l|}{ 7: frog } \\
\hline \multicolumn{2}{|l|}{ 8: frogs } \\
\hline 9: light & 9: light \\
\hline 10: lite & 10: lite \\
\hline \multicolumn{2}{|l|}{ 11: red } \\
\hline 12: the beer & 12: the beer \\
\hline 13: weed/beer & 13: weed/beer \\
\hline 14: weis & 14: weis \\
\hline 15: weiser & 15: weiser \\
\hline 16: wiser & 16: wiser \\
\hline
\end{tabular}

Figure 2. Sample of the program-generated Coder Comparison Report for prompted data. The records in this figure were randomly selected from a study of the effects of alcohol advertising. The numbers in parentheses next to Rater $A$ and Rater $B$ indicate the numbers of independently coded responses assigned to the designated category. The underlines in this table are hyperlinks back to the original coding screen for the respective items.

information about the coders and set up banks for each coder, (5) conduct the actual coding, (6) resolve coding discrepancies, and (7) generate and export the final dataset. Supervisors may access all of the functions, but coders have access to only the coding function. The following section briefly describes how to use the application, and step-by-step instructions are available at the help menu.

\section{Uploading and Setting Up the Coding Structure on the Server}

The upload dataset interface provides the user with a place to upload a dataset to be coded in CSV format to the server. After a dataset is uploaded to the system, the user will be able to specify more information related to this particular dataset including (1) coders' usernames and passwords; (2) the coding scheme, which may be an existing scheme already in the system or a newly developed scheme; (3) which coding schemes are to be associated with which variables in the uploaded dataset; (4) whether the coding should be with or without prompted cue words, with the default being "prompted"; (5) whether or not to use "autocoding" mode (under the default mode of autocoding, the same response from the same variable will be coded only once; if the autocoding mode is turned off, the response will always need to be coded, regardless of whether it has appeared and been coded in previous observations); and (6) whether to use multiple or single-category coding. By default, coders are allowed to code a response from zero to all categories. The user has the option to set this database-specific feature to limit the number of categories that can be coded to 1 , or any number up to the maximum number of categories in the coding scheme.

\section{Conducting the Actual Coding}

Coders log in, select the dataset and variable to be coded, and begin to code. The coder sees a screen with a list of the coding categories assigned to that variable along with the cue (prompt) in a column named Variable if the option to code with a visible cue is turned on. The subject's response is displayed at the top of the next column, named Value (see Figure 3). The coder categorizes the response by clicking the box next to the appropriate coding category (or categories). Coders may access detailed instructions on how to use the system at the help menu. New coders are provided with online training that explains how to execute the coding program.

\section{Resolving Coding Discrepancies and Examining Interrater Agreement}

Coding discrepancies may be examined at the resolve discrepancies interface. After a data structure is selected, all banks for that data structure will be listed in a bank box, and options are provided to select the banks and variables one wishes to examine. The user defines a consensus bank name or selects an already created consensus bank from a pull-down menu. The coding results in the selected banks 


\begin{tabular}{|c|c|c|}
\hline & Variable & Value \\
\hline Original & P3_SHOT & tequila \\
\hline 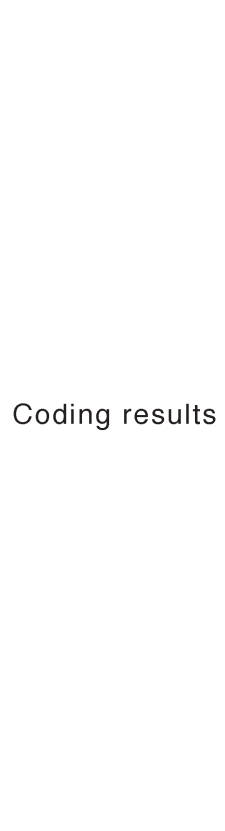 & $\begin{array}{r}\text { Alcohol: } \\
\text { Marijuana: } \\
\text { Amphetamine: } \\
\text { Hallucinogen: } \\
\text { Crack: } \\
\text { Heroin: } \\
\text { Tobacco: } \\
\text { Other_Non_Specific_Drug_Related: } \\
\text { Sex: } \\
\text { Food: } \\
\text { Exercise: } \\
\text { Violence: } \\
\text { Other_Definitely_Not_in_Other_Cat: } \\
\text { Other_Health_Related: }\end{array}$ & $\begin{array}{l}\square \\
\square \\
\square \\
\square \\
\square \\
\square \\
\square \\
\square \\
\square \\
\square \\
\square \\
\square \\
\square \\
\square \\
\square \\
\square\end{array}$ \\
\hline
\end{tabular}

Figure 3. Sample Web-based coding screen used by the coders assigning verbatim responses to categories. The response to be coded (which comes from the input stream) can appear for coder reference at the top of the screen. All categories of research interest are listed vertically down the left side of the screen. Verbatim responses viewed by the coders are assigned to the categories shown; a check mark in the box indicates that the response at the top of the screen matches the category.

are displayed column by column, and coding discrepancies are highlighted in red. A consensus coder resolves discrepancies.

The interrater agreement option may be selected from the main menu. The user will be guided to choose a dataset, choose the banks in which the coding results of different coders are to be stored, choose a scheme and the individual category (the default is all categories), and calculate the unadjusted and adjusted kappas for interrater agreement in each of the categories.

\section{Generating and Exporting the Final Dataset}

The user prepares a table for every bank associated with a particular data structure. The table export generator provides pull-down menus to select the data structure and bank. After clicking on the Export to a CSV File button, a CSV file will be generated and the user prompted to save the file into a designated directory on the local computer. The exported CSV file includes all the variables in the uploaded dataset, together with the coding results.

\section{DEMONSTRATION: STUDY 1}

\section{Method}

Participants. We tested the feasibility and efficacy of the coding application on a research study on alcohol use, linking associative responses to behavior. Participants were 1,761 ethnically diverse middle-school adolescents in southern California (mean age $=$ $12.47, S D=.91)$.

Procedure. In classroom settings, participants completed both surveys assessing various theoretical domains related to substance use and word association tasks. The adolescent participants in the alcohol study completed free and controlled association tasks (described in Stacy, 1997; see Table 1). The free association task used homographs (ambiguous words) as stimuli (e.g., "draft," "field"). The free association instructions asked respondents to "write next to each word the first word it makes you think of." Some of the words were drug-related homographs, and others were various miscellaneous words. Free association responses to the drug-related words have been used in the health behavior literature to measure cuebehavior associations - that is, the association in memory between ambiguous word cues and behaviors, such as alcohol or marijuana use (Stacy, 1997; Stacy, Ames, Sussman, \& Dent, 1996).

The controlled association task (Cramer, 1968) used very short phrases as stimuli (e.g., "feeling more relaxed") and asked respondents to "write the first behavior or action the phrase makes you think of." This controlled association task has been used to measure associations in memory between possible outcomes (e.g., relaxation) and behaviors (e.g., alcohol use) and has thus been classified as a measure of outcome-behavior associations in the health behavior literature (Ames \& Stacy, 1998; Stacy, 1997). Various forms of controlled association have been used more generally to obtain frequency norms applicable to different materials used in experimental work, such as category exemplars.

The participants were informed that their participation in the study was voluntary and that they could withdraw at any time 


\begin{tabular}{|c|c|c|c|c|}
\hline & Variable & \multicolumn{3}{|c|}{ Value } \\
\hline Original & P3_BUD & \multicolumn{3}{|c|}{ smoke } \\
\hline \multirow{17}{*}{ Coding results } & & Rater A & Rater B & Test \\
\hline & Alcohol: & & 0 & $\square$ \\
\hline & Marijuana: & & 1 & $\square$ \\
\hline & Amphetamine: & & 0 & $\square$ \\
\hline & Hallucinogen: & & 0 & $\square$ \\
\hline & Crack: & & 0 & $\square$ \\
\hline & Heroin: & & 0 & $\square$ \\
\hline & Tobacco: & & 0 & $\square$ \\
\hline & Other_Specific_Drug_Related: & & 0 & $\square$ \\
\hline & Other_Non_Specific_Drug_Related: & & 0 & $\square$ \\
\hline & Sex: & 0 & 0 & $\square$ \\
\hline & Food: & & 0 & $\square$ \\
\hline & Exercise: & & 0 & $\square$ \\
\hline & Violence: & 0 & 0 & $\square$ \\
\hline & Other_Health_Related: & & 0 & $\square$ \\
\hline & Other_Definitely_Not_in_Other_Cat: & & 0 & $\square$ \\
\hline & Does_Not_Fit: & 0 & 0 & $\square$ \\
\hline
\end{tabular}

Figure 4. Example of the Web-based coding screen provided for the consensus coder. The consensus coder checked a box in the test column to choose a coding for the subject's response (shown in the row below the value label) as the appropriate consensus code.

without prejudice. The data collection procedure was anonymous; guarantees of anonymity have been found to lead to more valid responses in drug surveys (e.g., Murray \& Perry, 1987; Stacy, Widaman, Hayes, \& DiMatteo, 1985).

Trained research assistants used the coding application to assign each unique response to a predefined behavioral category. ${ }^{2}$ At any given time, two coders were assigned to categorize a set group of verbatim responses. In the alcohol study, the adolescents' responses to ambiguous alcohol-related cues (e.g., "tap," "draft," "pitcher," "bud," "hammered," "shot") and ambiguous phrases (e.g., "having fun," "feeling good," "feeling more relaxed") were coded (cued words and phrases are listed in Table 1). For this study, we chose to focus our coding efforts on prompted data. Each task (e.g., responses to ambiguous words or phrases) and all responses to a single cue word or phrase were coded separately, and each response was coded by two independent coders.

\section{Results}

Coding of the adolescents' responses on the word association tasks showed almost perfect interrater agreement for items assigned to the alcohol category (with visible

Table 1

Interrater Reliability for the Free Association and Controlled Association

Tasks for Responses Assigned by Two Coders to the Alcohol Category

\begin{tabular}{lccc}
\hline & $\begin{array}{c}\text { No. of Responses } \\
\text { Coded }\end{array}$ & Kappa & Adjusted Kappa \\
\hline Free Association Prompt & & & \\
Tap & 127 & .853 & .984 \\
Draft & 137 & 1.000 & 1.000 \\
Hammered & 115 & .786 & .948 \\
Pitcher & 83 & 1.000 & 1.000 \\
Shot & 97 & .942 & .979 \\
Bud & 80 & .945 & .975 \\
Controlled Association Prompt & & & \\
Pleasant times & 269 & .663 & .985 \\
Feeling more relaxed & 244 & .796 & .984 \\
Having fun & 240 & .746 & .983 \\
Feeling happy & 294 & .743 & .973 \\
Having a good time & 214 & .741 & .963 \\
Being cool & 321 & .887 & .994 \\
Forgetting problems & 285 & .885 & .986 \\
Being more social & 241 & .794 & .975 \\
Feeling good & 267 & .764 & .978 \\
Taste something good & 214 & .395 & .972 \\
Feeling buzzed & 219 & .964 & .973 \\
\hline
\end{tabular}

Note-Kappas were based on uniquely coded responses (i.e., automatically coded repetitions are not included in the calculation of kappa) and prompted data only. 
prompts), as revealed by the average adjusted kappa coefficients across items on the free association task (kappa $=$ 0.975 ) and on the controlled association task (kappa $=$ 0.979; see Landis \& Koch, 1977, for interpretation of kappa). The reported kappas were based on the unique coded response for a given cue word or phrase and did not include autocoded repetitions (see Table 1).

\section{DEMONSTRATION: STUDY 2}

\section{Method}

Participants. In the next study, we again tested the efficacy of the coding application on a research study on alcohol and other drug use, linking associative responses to behavior. Participants were 58 adolescents in southern California continuation high schools (mean age $=16.08, S D=1.4$ ). The youth in continuation high schools have transferred from the regular/comprehensive school system for various reasons, including substance use, and they are at relatively high risk for substance abuse (see Sussman, Dent, et al., 1995; Sussman, Stacy, et al., 1995).

Procedure. As in the first demonstration study, participants completed surveys with a variety of measures related to substance use and word association tasks. For the present demonstration, we coded the participants' verbatim responses on a compound cue task using a health behavior scheme. The compound-cue task consisted of a combination of cues (e.g., "my bedroom/having fun") taken from the controlled association task discussed above, and a global situations task, consisting of ambiguous situations. The situations used in the compound cue task were those highest frequency situations determined in a prior study (e.g., "my bedroom"; see Sussman, Stacy, Ames, \& Freedman, 1998) to be high risk by continuation high school youth. In general, memory research presents a single cue to elicit a target concept, but this may be unrealistic given the complex context provided for retrieval in real-world events (see Dosher \& Rosedale, 1997). Therefore, in our studies of drug use behavior, we investigate compound cues and the effect of multiple cues on memory activation (see Table 2 for compound-cue items). In many instances, it is only when a cue is coupled with a second cue that its power as a "trigger" of relevant cognitions is detected. That is, the two cues form a constellation that is more than the sum of its parts (for discussion, see Dosher \& Rosedale, 1997).

Participant-generated responses to these compound cues were entered verbatim into computer files by an independent data entry firm, and verbatim responses were coded by two independent coders into health behavior categories similar to those shown in Figure 3. In order to determine whether providing the coder with the context in which a response was made (i.e., the question to which the student was responding) assisted the coder in deciding to which behavior category to assign the response, we assessed coder agreement when the stimulus cues were either visible ("prompted") or not visible to the rater ("unprompted") during the coding process and compared the level of agreement under these two conditions (see Table 2). For prompted data, if the cue phrase was "my bedroom/having fun," then all responses to "my bedroom/having fun" were coded separately from responses to cued words such as "friend's house/having fun." Coding the responses in the context of the prompt meant that multiple instances of the same response across cued words needed to be coded, but multiple instances for a single cue did not. This approach increases the time required for coding, but, depending on the dataset, may provide more statistically reliable results overall.

\section{Results}

The coding of participant-generated responses on the compound cue task showed almost perfect interrater agreement for items assigned to the alcohol, marijuana, sex, and drug effect categories. In addition, the adjusted kappa coefficients across items on the compound-cue task

Table 2

Interrater Reliability (Adjusted Kappa) for Responses Assigned by Two Coders to Four Coding Categories When the Data Were Presented to the Coders With and Without Prompts

\begin{tabular}{|c|c|c|c|c|c|}
\hline \multirow[b]{2}{*}{ Compound-Cue Task } & \multirow{2}{*}{$\begin{array}{c}\text { Number of } \\
\text { Responses Coded }\end{array}$} & \multicolumn{4}{|c|}{ Coding Category Agreement Kappa } \\
\hline & & Alcohol & Marijuana & Sex & Drug Effects \\
\hline \multirow[t]{2}{*}{ My bedroom/Having fun } & 49 & 1.00 & 1.00 & .84 & .96 \\
\hline & & 1.00 & 1.00 & .88 & 1.00 \\
\hline \multirow[t]{2}{*}{ Friend's house/Feeling relaxed } & 48 & 1.00 & 1.00 & .92 & 1.00 \\
\hline & & .96 & 1.00 & .92 & .79 \\
\hline \multirow[t]{2}{*}{ In my yard/Feeling relaxed } & 53 & 1.00 & .96 & .93 & 1.00 \\
\hline & & 1.00 & .93 & 1.00 & .81 \\
\hline \multirow[t]{2}{*}{ My bedroom/Feeling good } & 39 & .95 & 1.00 & .85 & .80 \\
\hline & & 1.00 & 1.00 & .90 & .95 \\
\hline \multirow[t]{2}{*}{ In my yard/Feeling good } & 51 & .96 & .96 & .88 & .88 \\
\hline & & 1.00 & 1.00 & 1.00 & .80 \\
\hline \multirow[t]{2}{*}{ Friend's house/Having fun } & 39 & 1.00 & 1.00 & .95 & .95 \\
\hline & & 1.00 & 1.00 & 1.00 & 1.00 \\
\hline \multirow[t]{2}{*}{ In my yard/Feeling happy } & 47 & 1.00 & .96 & .87 & .87 \\
\hline & & .96 & .96 & .92 & .83 \\
\hline \multirow[t]{2}{*}{ My bedroom/Feeling relaxed } & 40 & 1.00 & 1.00 & .80 & .90 \\
\hline & & 1.00 & 1.00 & .85 & .90 \\
\hline \multirow[t]{2}{*}{ Friend's house/Feeling good } & 47 & .96 & .96 & .83 & .92 \\
\hline & & .96 & 1.00 & .83 & .79 \\
\hline \multirow[t]{2}{*}{ Mean adjusted kappa } & & .99 & .98 & .87 & .92 \\
\hline & & .99 & 1.99 & .92 & .87 \\
\hline
\end{tabular}

Note-Kappas were based on uniquely coded responses (i.e., automatically coded repetitions are not included in the calculation of kappa). The first value presented in each cell is the agreement for unprompted data, and the second, for prompted data. 
were the same or almost the same for responses coded when the prompt was visible and when responses were coded without prompts. The interrater agreement across items assigned to the alcohol category was almost perfect (mean adjusted kappa $=.99$ ) for both prompted and unprompted coded responses. For the marijuana category, the unprompted average kappa coefficient (kappa $=.98)$ and the prompted average kappa coefficient (kappa $=$ .99) were similar. For the drug effects category, the unprompted average kappa coefficient (kappa $=.92)$ was slightly higher than the prompted average kappa coefficient (kappa $=.87$; see Table 2 for interrater reliability for responses on the compound cue task).

\section{CONCLUSIONS}

The present Web-based coding system for behavior research described in this article expands on previously developed applications for verbal report data. This system effectively reduces many of the drawbacks to coding open-ended responses and provides a reasonably simple way to ensure quality control and statistical reliability. Because the system is Web based, multiple coders can work from any Internet-enabled computer when assigning open-ended responses to predetermined categories. In addition, the various functions (e.g., inclusive quality control processes, prompted vs. unprompted coding options, easy definition of coding schemes, and the automatic coding of repetitions option) make this Webbased system quite flexible for use by most researchers interested in coding participant-generated responses. By choosing to automatically assign repeated responses to a category once a response has initially been coded to the same category (where appropriate), the coding program diminishes the amount of time it takes to code the data, particularly among datasets in which similar responses are prevalent. And, although time must be spent to train anyone who will use the program, the instruction process is relatively short; coders should be able to begin work on the dataset the same day they are introduced to the coding program. Our findings show that research assistants with minimal training are able to reliably code participantgenerated responses to word association tasks with the use of this coding system. Statistical reliability is also assured by the inclusion of quality control procedures.

In the studies discussed in this article, the coders initially evaluated participants' responses to the open-ended tasks without knowing the cues that prompted the responses. In the second demonstration study, interrater agreement and coding accuracy were fairly consistent between coding with visible cues that prompted the responses and coding without the visible cues. However, coding agreement was not as consistent in the first demonstration study between coding with and without visible cues. Cues provide an important level of context for interpretation of responses. For the first demonstration study, when coding responses without any context (i.e., without a visible cued word or phrase on the coding screen), the various word meanings associated with ambiguous words (i.e., the primary stimuli for implicit cognition work) resulted in some words being inaccurately or inconsistently assigned to categories. For example, the response word "hammered" to the cue word "nail" is very different from that of "draft." The response word "hammered" to the cue word "draft" is a likely behavior associate of alcohol use, whereas "hammered" in response to the cue word "nail" is not.

In our research, many stimulus words are chosen to provide cues that implicitly activate memory associations or responses related to repeatedly performed behaviors (e.g., alcohol use, marijuana use, etc.) that make ambiguous words salient. Without presentation of the stimulus words during the coding of responses, it is possible for the response words to be inaccurately coded. Because we were using the coding program's option to automatically code identical repetition responses once a coder made a decision, the problem of "context" was exacerbated in the first demonstration study. For instance, assume the response "wiser" occurs several times in a dataset. Viewing the data without the survey items presented at the time of coding, a coder might assume that "wiser" does not belong in any of the defined behavioral categories. The coder would not know that that instance followed the cue word "bud," marking it for placement in the alcohol category.

Alternatively, there are times when the researcher might choose to code only unprompted data in order to avoid any bias resulting from exposure to the stimulus words. The presence of a prompt could bias independent raters in the same way, resulting in category assignment responses being more consistent across raters. This would inflate the measure of reliability; that is, the raters may agree more frequently than they would otherwise when provided a context for the response being coded. Without the prompt, the raters' judgments about category assignments might be more variable. There are trade-offs in coding with visible prompts and coding when the prompts are not visible. If the researcher chooses to code responses with visible prompts, an independent assessment of category validity is encouraged. One example of a situation in which it is preferable to code responses without visible stimulus words would be that in which cues are manipulated across survey forms. In this situation, in order not to bias the effect of the manipulation, coders should not be aware of the cues when coding responses.

Although the studies discussed as examples in this article involved health behavior data, the present Web-based coding system is flexible enough so that its advantages can be applied to the coding of a wide range of different types of participant-generated responses. This application is useful in both experimental and survey research. Variables, categories, and the coding screen itself can be changed at the program level to accommodate many types of studies. The ease in coding and the confidence in the data that the coding system allows may benefit future research on association norms in large samples in cogni- 
tive research (e.g., Nelson, McEvoy, \& Schreiber, 1998) and survey research using participant-generated or openended methods.

\section{REFERENCES}

Ames, S. L., \& Stacy, A. W. (1998). Implicit cognition in the prediction of substance use among drug offenders. Psychology of Addictive Behaviors, 12, 272-281.

Bishop, G. F., Tuchfarber, A. J., \& Oldendick, R. W. (1986). Opinions on fictitious issues: The pressure to answer survey questions. Public Opinion Quarterly, 50, 240-250.

Bornstein, R. F. (1999). Criterion validity of objective and projective dependency tests: A meta-analytic assessment of behavioral prediction. Psychology Assessment, 11, 48-57.

Carey, J., Wenzel, P., Reilly, C., Sheridan, J., \& Steinberg, J. (1998). CDC EZ-Text: Software for management and analysis of semistructured qualitative data sets. Cultural Anthropology Methods, 10, 14-20.

Cramer, P. (1968). Word association. New York: Academic Press.

CRUTCHER, R. (2003). A computer-aided digital audio recording and encoding system for improving the encoding of verbal reports. Behavior Research Methods, Instruments, \& Computers, 35, 263-268.

Dosher, B. A., \& Rosedale, G. S. (1997). Configural processing in memory retrieval: Multiple cues and ensemble representations. Cognitive Psychology, 33, 209-265.

JAMES, J., \& SANDERSON, P. (1991). Heuristic and statistical support for protocol analysis with SHAPA Version 2.01. Behavior Research Methods, Instruments, \& Computers, 23, 449-460.

Krosnick, J. A. (1999). Survey research. Annual Review of Psychology, 50, 537-567.

Landis, J. R., \& Косн, G. G. (1977). The measurement of observer agreement for categorical data. Biometrics, 33, 159-174.

Murray, D. M., \& Perry, C. L. (1987). The measurement of substance use among adolescents: When is the "bogus pipeline" method needed? Addictive Behaviors, 12, 225-233.

Nelson, D. L., McEvoy, C. L., \& Schreiber, T. A. (1998). The University of South Florida word association, rhyme, and word fragment norms. www.usf.edu/FreeAssociation/.

STACY, A. W. (1997). Memory activation and expectancy as prospec- tive predictors of alcohol and marijuana use. Journal of Abnormal Psychology, 106, 61-73.

Stacy, A. W., Ames, S. L., Sussman, S., \& Dent, C. W. (1996). Implicit cognition in adolescent drug use. Psychology of Addictive Behaviors, 10, 190-203.

Stacy, A. W., Widaman, K. F., Hayes, R., \& DiMatteo, M. R. (1985). Validity of self-reports of alcohol and other drug use: A multitraitmultimethod assessment. Journal of Personality \& Social Psychology, 49, 219-232.

Sussman, S., Dent, C. W., Simon, T. R., Stacy, A. W., Galaif, E. R., Moss, M. A., ET AL. (1995). Immediate impact of social influenceoriented substance abuse prevention curricula in traditional and continuation high schools. Drugs \& Society, 8, 65-81.

Sussman, S., Stacy, A. W., Ames, S. L., \& Freedman, L. B. (1998). Self-reported high-risk locations of adolescent drug use. Addictive Behaviors, 23, 405-411

Sussman, S., Stacy, A. W., Dent, C. W., Simon, T. R., Galaif, E. R., Moss, M. A., ET AL. (1995). Continuation high schools: Youth at risk for drug abuse. Journal of Drug Education, 25, 191-209.

Verbastat 7.o [Computer software]. (2004). Chicago: SPSS. www .spss.com/verbastat/.

WALONICK, D. (2004). StatPack [Computer software]. Minneapolis: Author. www.statpac.com.

White, D., King, A., \& Duncan, S. (2002). Voice recognition technology as a tool for behavioral research. Behavior Research Methods, Instruments, \& Computers, 34, 1-5.

\section{NOTES}

1. The source code and documentation are freely available to interested users; however, if technical support is needed, there may be a consulting fee.

2. To date, 12 coders have been trained and have used the Web-based coding application on four different projects. Several more coders have been trained on a precursor (non-Web-based) version of this coding system, which has been used for several projects at two institutions.

(Manuscript submitted April 24, 2003;

revision accepted for publication December 3, 2004.) 